\title{
Anaesthetic implications during the COVID-19 pandemic: an appraisal of the literature
}

\author{
Ravindri Jayasinghe ${ }^{1}$, Nadeeshya Welikala $^{2}$,Ravindi Gunaratne ${ }^{2}$, Umesh Jayarajah ${ }^{1}$,Vihara Dassanayake ${ }^{1}$ \\ Sanjeewa Seneviratne ${ }^{1}$ \\ ${ }^{1}$ Department of Surgery and Department of Anaesthesiology \& Critical Care, Faculty of Medicine, \\ University of Colombo, Sri Lanka \\ ${ }^{2}$ Department of Anaesthesiology \& Critical Care, National Hospital Colombo, Sri Lanka
}

Keywords: Anaesthesia; anaesthesiology; COVID-19; pandemic; SARS-CoV-2

\begin{abstract}
The coronavirus disease-2019 (COVID-19) pandemic has raised many concerns regarding the safety of patients and healthcare workers. Anaesthetic implications and the impact of this pandemic on anaesthesiologists have been discussed widely in the recent past. Operating theatres are high-risk areas where anaesthesiologists perform various aerosolgenerating procedures (AGPs).
\end{abstract}

Prolong patient contact predisposes them to the risk of exposure. Scarcity of resources and limitations in testing pose an additional risk to the anesthesiologist. Although guidance from various professional bodies is in abundance, there is a general lack of consensus. This review summarises current evidence and guidelines related to anaesthesiology during the COVID-19 pandemic. Ensuring the provision of unhindered care, ensuring patient safety and protecting oneself with optimum utilization of available resources remain a challenge to the anesthesiologist.

\section{Introduction}

SARS-CoV-2, a novel strain of coronavirus was first isolated in January 2020 while investigating an outbreak of pneumonia in Wuhan, China [1]. Since its origin, millions of cases have been confirmed positive and more than 2.5 million deaths have been reported worldwide [2]. The pandemic has had a significant impact on the healthcare system, economy and the general public $[3,4]$.

The impact of COVID-19 on the anaesthesiologist is considerably higher when compared to other healthcare workers due to their involvement in airway management in a high-risk environment [5]. Hospitals worldwide have taken measures to reduce the number of procedures to minimise disease transmission and to preserve both consumables and human resources [6]. Rational use of personal protective

Correspondence: Umesh Jayarajah

E-mail: umeshe.jaya@gmail.com

(i) https://orcid.org/0000-0002-0398-5197

Received: 21-03-2021 Accepted: 30-03-2021 DOI: http://doi.org/10.4038/sljs.v39i1.8814 equipment (PPE) is essential to containing the spread of infection. The objective of this review is to focus on the current practice of anaesthesiology during the pandemic and to summarise key recommendations based on consensus guidelines for anaesthesiologists.

Mode of anaesthesia: General anaesthesia (GA) vs. Regional anaesthesia (RA)

Anaesthesia for patients with COVID-19 mandates a balance between the provision of care and safety for both the patient and the anesthesiologist. However, during the process of shared decision- making, the priority is to provide the safest and the most appropriate mode of anaesthesia for a surgical procedure to the patient. Whenever there is a doubt, patient comfort and safety should be considered as a priority. The consenting process should not be biased towards any particular modality and all information based on evidence should be provided to the patient. It is also prudent to consider the risks of disease transmission related to the mode of anaesthesia and the urgency of surgery [7].

Many potential advantages have been described concerning various modes of anaesthesia. Regional anaesthesia reduces the number of AGPs thereby saving time and cost spent on PPEs, preservation of immune function when compared with general anaesthesia, improved postoperative analgesia minimising direct contact with caregivers, and earlier hospital discharge [8]. Additionally, it has been identified that patients with acute COVID-19 infection were at an increased risk of developing postoperative pulmonary complications including ARDS [9, 10]. Overall, patients who underwent neuraxial anaesthesia had fewer perioperative pulmonary complications compared to general anaesthesia [11].

To date, no published randomized controlled trials are evaluating the reduction of risk for disease transmission brought on by regional anaesthesia when compared to general anaesthesia. Therefore, it is reasonable to consider that all the recommendations on the above have been based on logical thinking and understanding. Interestingly, a recent death review of health care workers carried out in the United Kingdom revealed that there were no reported deaths among anaesthesiologists or staff involved in critical care practices, 
where the highest number of AGPs had been carried out [8]. The reason for this is unclear but may well be attributed to the rigorous utilization of PPEs [8].

Based on the above, regional anaesthesia may be considered a suitable option where appropriate. However, the risk of conversion to GA midway during the procedure should be thoroughly evaluated beforehand and appropriate PPEs should be kept ready. It should be remembered that droplet and aerosol generation are possible while breathing, speaking, coughing and sneezing which are all possible in the awake patient under regional anaesthesia. In such patients, donning in only droplet PPEs may be sufficient. Such a situation may not arise or be minimal in a patient who is under general anaesthesia with a definitive airway in situ, which might appear to be a safer option as opposed to regional anaesthesia.

Other general standards should be given due consideration such as limiting proximity to the patient and duration of exposure, minimum of five air changes/hour, placement of a surgical mask on the patient and minimal manipulation of oxygen therapy devices. Avoidance of high oxygen flow rates will minimize the generation of aerosols.

Furthermore, with more cases being performed under regional anaesthesia there are advantages to an institution, such as efficient utilization of drugs for critical care services, reducing the time consumed per case and bypassing the time spent in the recovery area.

Due to a lack of reliable evidence, the adverse effects of regional anaesthesia on COVID-19 patients have not been fully demonstrated. Literature suggests there are minimal effects on clinical outcomes in patients undergoing regional anaesthesia [11].

Despite being commonly associated with hypercoagulability, COVID-19 is also associated with thrombocytopenia [12]. Therefore, a full blood count is mandated before providing neuraxial anaesthesia. Care needs to be taken during the insertion and removal of indwelling epidural catheters with pre-existing coagulopathy.

In patients with neurological disease, central neuraxial blocks (CNB) were thought to be safe as the coronavirus had not been isolated in cerebrospinal fluid [8]. However, with a more recently reported case of COVID-19 meningitis and detectable virus within the CSF, such evidence should be revisited [8].

During placement of peripheral nerve blocks, several key recommendations have been made by various bodies to improve quality and safety related to anaesthesia. Use of ultrasonography to perform regional blocks, use of wound infiltration catheters in case of prolonged blocks or to provide post-operative pain relief using adjuncts to peripheral nerve blocks (PNB) along with local anaesthetics without dose modifications, can all be incorporated into current practise during the pandemic [8].

Accredited authorities of regional anaesthesia had issued guidance on neuraxial anaesthesia and peripheral nerve blocks during the COVID-19 pandemic recommending regional anaesthesia over general anaesthesia [13]. However, the possibility of a "failed block" and conversion to general anaesthesia may increase the risk of viral transmission. Therefore, careful preoperative assessment to avoid any unplanned conversions to general anaesthesia is imperative.

\section{Pre-operative assessment and Preparation}

During the preoperative assessment, it is important to weigh the risk of disease transmission and the benefit of surgery to the patient. Identifying the high-risk patient is of paramount importance [14]. It is also important to balance resource consumption and the risk of transmission of the disease to healthcare workers. Factors such as the need for critical care beds, transfusions, number of personnel required in theatre, risks associated with various modes of anaesthesia and type of surgery should all be taken into consideration $[15,16]$. It is recommended to have a designated operating room for COVID-19 suspected patients and to do with the appropriate level of PPEs. Limited resources including consumables is a universal problem. The World Health Organization (WHO) recommends using N95, Filtering Face Piece-2 (FFP2) respirator or an equivalent during aerosol-generating procedures [17]. Despite guidance on PPEs, there is limited consensus related to their usage. A three-tiered approach to PPE has been recommended for airway managers. Their recommendation is to use airborne, droplet and contact precautions in combination for high-risk aerosol-generating procedures which comprise of a head cover, eye protection, N95 respirator, AAMI (Association for the advancement of medical instrumentation) level-2 or higher gown and a pair of single gloves overlapping the sleeve of the waterproof gown [17]. Droplet and contact precautions are considered adequate for most routine procedures. High-risk precautions should be used by personnel directly involved in procedures such as airway management. Limiting the number of personnel in the operating room (OR) is critical during high-risk procedures which minimises exposure [17].

It is essential to have a pre-planned team led by a senior anaesthesiologist for patients suspected or confirmed with COVID-19. Roles should be allocated preoperatively, and all members should maintain closed-loop communication. During emergency surgery, a member of the team should perform a rapid pre-anaesthetic check-in in a predesigned room within the theatre [18]. During an elective procedure, 
pre-anaesthetic patient assessment could be done via telemedicine as an alternative to face-to-face consultation. Video and audio devices can be used to perform a visual examination and provide medical advice. Most routine surgeries including cardiac, vascular and thoracic surgery can potentially be performed following a telemedicine evaluation. However, there are limitations especially in patients with multiple comorbidities and technological constraints related to internet access and the level of literacy. This is a hindrance and a constant challenge to the anesthesiologist and patients seeking perioperative care in a low-middle income country, such as Sri Lanka.

\section{Airway management}

Consensus guidelines for managing the airway in patients with COVID-19 was published by the Difficult Airway Society, the Association of Anaesthetists, the Intensive Care Society, the Faculty of Intensive Care Medicine, and the Royal College of Anaesthetists [19].

Airway establishment and management may be required for asymptomatic or minimally symptomatic patients with COVID 19 in the community requiring emergency surgery for unrelated conditions [19] and controlled ventilation for patients diagnosed with viral pneumonia in the critical care unit.

Several AGPs have been identified as high-risk of dissemination of the virus during airway manipulation. These include tracheal intubation, tracheostomy, emergency frontof neck airway (FONA), non-invasive ventilation (NIV), and bag-mask ventilation. Other possible AGPs includes tracheal extubation, disconnection of ventilatory circuits during use, performing chest compressions during cardiopulmonary resuscitation without tracheal intubation (minimal risk during defibrillation), bronchoscopy, and tracheal suctioning.

Recent studies have demonstrated that in practice, tracheal intubation and facemask ventilation produced only very low quantities of aerosolised particles. Tracheal extubation when associated with coughing produced 15 times more aerosols than intubation. Volitional coughing is associated with a very high aerosol generation [20].

To ensure safety for the anesthesiologist and other health care workers, correct use of PPEs, decontamination of surfaces and equipment, minimizing unnecessary patient and surface contact and careful waste disposal are essential elements during patient care. Double gloving may protect against fomite contamination. Training and regular simulation sessions in the use of PPEs (both donning and doffing) before patient management is beneficial [19].
To minimize the risk of airborne exposure, it is best to manage the patient in a negative pressure room with rates of air exchange amounting to more than 12 exchanges per hour. Monitoring should be established by adhering to the Association of Anaesthetists standards including the use of continuous waveform capnography.

Intubation should be performed by the most experienced member of the team to ensure success at the first attempt, safety, accuracy and swiftness in securing the airway. A tracheal intubation trolley must be prepared and be checked daily. An airway strategy including rescue plans should be briefed to the airway team before intubation. The team should comprise of an "intubator", an assistant and a third staff member to administer drugs and to monitor the patient. A runner or buddy should be present outside the intubating bay who can call for help if required and assist in donning of team members and observe doffing once the procedure is over.

Rapid sequence induction (RSI) with cricoid pressure is recommended to secure the airway. The procedure begins with pre-oxygenation using a well-fitting mask for 3-5 min with a closed circuit. A heat and moisture exchange (HME) filter should be connected between the catheter mount and the circuit. Non-invasive ventilation and high flow nasal oxygen should be avoided. Ketamine can be used as an induction agent to avoid cardiovascular collapse provided that there is no contraindication. Rocuronium (if sugammadex is available) or succinylcholine is used as neuromuscular blockers and complete paralysis must be confirmed before attempting intubation. Face mask ventilation should be avoided during apnoea. Continuous positive airway pressure (CPAP) may be applied. If mask ventilation is necessary to prevent hypoxemia, 2-hand, 2-person technique with a VE grip should be used.

The use of a video-laryngoscope is advocated as the operator can maintain sufficient distance from the patient's airway during tracheal intubation and achieve prompt first-pass tracheal intubation. The tracheal tube has to be placed without losing sight of it on the screen and the cuff is passed $1-2 \mathrm{~cm}$ below the cords for optimal positioning. The cuff is inflated to 20-30 cmH2O. Mechanical ventilation is commenced only after cuff inflation. Correct placement of the tracheal tube is then confirmed with continuous waveform capnography. Auscultation to confirm tube placement is not recommended. Visual observation of equal bilateral chest wall expansion, lung ultrasound or chest X-ray can also be done to confirm the correct placement of the tube.

A ventilator dedicated for COVID-19 positive patients in the OR has also been recommended which would minimize cross-contamination [21]. The use of barrier-enclosure 
systems, such as aerosol boxes and plastic drapes have been developed to act as a shield during endotracheal intubation while allowing enough mobility during intubation. Its use may complicate airway management with potential adverse events, particularly during airway emergencies. Concerns include the limited ability for interventions, limited access to support during intubation by the assistant, damage to the PPE of the "intubator", injuries to the patient and secondary aerosolization during barrier removal. There is no clear evidence to suggest additional protection for HCWs and there is no standard for cleaning such equipment. Enclosure barriers cannot substitute adequate use of PPEs and further studies are required for validation [22].

\section{Perioperative management and extubation}

Adherence to the prevention of infections is mandatory during the intraoperative period. As a policy for infection control, it is recommended that only one anesthesiologist should use the workstation and it should be covered with disposable transparent drapes. Frequent intraoperative suctioning should be avoided and low-flow anaesthesia should be used for maintenance to reduce viral transmission [18].

Tracheal extubation should be performed inside the operating theatre with limited HCWs and efforts taken to minimize coughing due to increased aerosol generation. Use of supraglottic airway as a bridging technique or use of medications such as dexmedetomidine, lidocaine and opioids which suppresses cough may be considered to achieve smooth extubation. However, the safety of these procedures in the setting of the COVID-19 pandemic has not been proven. Tracheal suctioning through a closed suction apparatus and oral suctioning can be performed as usual. There should be equipment available to provide low flow nasal oxygen $(<5$ litres/ min) or face mask oxygen before extubation to ensure the immediate connection of the patient to their oxygen mask or nasal cannula [19]. If extubation is deferred, the patient transfer should take place using a transport ventilator with an HME (heat and moisture exchanger) filter connected to the patient end [18].

\section{Recovery and post anaesthesia care}

Patients should be taken to a designated recovery room following extubation. The patients should wear surgical masks before leaving the theatre. Congestion in the recovery area should be avoided and experts recommend maintaining at least a one-meter distance between patients [23]. After leaving the theatre, it is recommended to keep the patient in an isolated room. However, due to overwhelmed resources, this may be impractical in many centres.
All used PPE s should be disposed into biohazard bags keeping with the local infection control policy. It is mandatory to avoid contamination during the doffing of PPEs. This could be achieved by having an observer/buddy to guide safe doffing [24]. Decontamination of the theatre and equipment should follow hospital policy. All clinical waste should be clearly labelled, double bagged and sealed. It is then sprayed with chlorinated disinfectant and covered with an additional bag. Single-use laryngoscopy blades, breathing circuits, masks, HME filters, tracheal tubes, gas sampling lines and soda-lime needs to be discarded [25]. Agents that are used for decontamination include, vaporized Glutaraldehyde (ANIOS) or hydrogen peroxide generator (VHPG), 1\% sodium hypochlorite solution, and 75\% alcohol. Metallic equipment must be kept immersed in $1 \%$ sodium hypochlorite for 30 minutes and then washed and wiped [25]. The operating theatre should be cleaned and disinfected between surgeries. The disinfecting personnel must enter the theatre only after adequate air changes have been completed to remove infectious particles. Staff must be aware of the COVID status of the patient and must handle equipment only after wearing full PPE [26]. All floors and walls should be cleaned with $1 \%$ sodium hypochlorite solution [25]. The theatre can be disinfected with UV light after chemical disinfection [26].

\section{Conclusion}

The ongoing pandemic is causing a dramatic shift in the role of healthcare workers in their diverse specialities. Anaesthesiologists are placed at high risk due to manipulation of the airway and dealing with AGPs. Hospitals should develop a detailed pandemic preparedness plan addressing major concerns such as limitation of resources, staff wellbeing and safety, and implementation of strict measures of infection control. Current guidance is mostly based on expert opinion. High-quality multicentre research is deemed essential to formulate evidence-based healthcare policy changes both locally and internationally.

All authors disclose no conflict of interest. The study was conducted in accordance with the ethical standards of the relevant institutional or national ethics committee and the Helsinki Declaration of 1975, as revised in 2000 .

\section{References}

1. Borges do Nascimento IJ, Cacic N, Abdulazeem HM: Novel Coronavirus Infection (COVID-19) in Humans: A Scoping Review and Meta-Analysis. 2020, 9(4):941

https://doi.org/10.3390/jcm9040941

2. Borges do Nascimento IJ, von Groote TC, O'Mathúna DP et al. Clinical, laboratory and radiological characteristics and outcomes of novel coronavirus (SARS-CoV-2) infection in humans: A systematic review and series of meta-analyses. PloS 
one 2020, 15(9): 0239235 .

https://doi.org/10.1371/journal.pone.0239235

3. Jayasinghe R, Ranasinghe S, Jayarajah U, Seneviratne S: Quality of online information for the general public on COVID-19. Patient Education and Counseling 2020 in press. https://doi.org/10.1016/j.pec.2020.08.001

4. Wijerathne PK, Nanayakkara K, Basnayake O, Gunapala N, Jayarajah U: Impact of COVID-19 on postgraduate surgical training: the trainees' perspective. Sri Lanka Journal of Surgery 2020, 38(3):53-55. http://doi.org/10.4038/sljs.v38i3.8769

5. Preventing infection of patients and healthcare workers should be the new normal in the era of novel coronavirus epidemics [https://anesthesiology.pubs.asahq.org/article.aspx?articleid=27 63452]

6. Collaborative C: Delaying surgery for patients with a previous SARS-CoV-2 infection. The British journal of surgery 2020, 107:e601-e602.https://dx.doi.org/10.1002\%2Fbjs. 12050

7. Glasbey JC, Bhangu A, Collaborative C: Elective cancer surgery in COVID-19-free surgical pathways during the SARS-CoV-2 pandemic: an international, multicenter, comparative cohort study. Journal of Clinical Oncology 2020:JCO. 20.01933. doi: https://doi.org/10.1101/2020.12.15.20248195

8. Macfarlane AJ, Harrop-Griffiths W, Pawa A: Regional anaesthesia and COVID-19: first choice at last? British journal of anaesthesia 2020, 125(3):243-247. https://doi.org/10.1016/j.bja.2020.05.016

9. Myles PS, Maswime S: Mitigating the risks of surgery during the COVID-19 pandemic. The Lancet 2020, 396(10243):2-3. https://doi.org/10.1016/S0140-6736(20)31256-3

10.Collaborative C: Preoperative nasopharyngeal swab testing and postoperative pulmonary complications in patients undergoing elective surgery during the SARS-CoV-2 pandemic. British Journal of Surgery. https://doi.org/10.1093/bjs/znaa051

11.Rodgers A, Walker N, Schug S, McKee A, Kehlet H, Van Zundert A, Sage D, Futter M, Saville G, Clark T: Reduction of postoperative mortality and morbidity with epidural or spinal anaesthesia: results from overview of randomised trials. Bmj 2000, 321(7275):1493.

https://doi.org/10.1136/bmj.321.7275.1493

12.Rahman A, Niloofa R et al. Hematological Abnormalities in COVID-19: A Narrative Review. The American journal of tropical medicine and hygiene:tpmd201536. https://doi.org/10.4269/ajtmh.20-1536

13.Practice Recommendations on Neuraxial Anesthesia and Peripheral Nerve Blocks during the COVID-19 Pandemic [https://www.asra.com/page/2905/practice-recommendationson-neuraxial-anesthesia-and-peripheral-nerve-blocks-dur]

14.Prachand VN, Milner R, Angelos P, Posner MC, Fung JJ, Agrawal N, Jeevanandam V, Matthews JB: Medically-necessary, timesensitive procedures: a scoring system to ethically and efficiently manage resource scarcity and provider risk during the COVID-19 pandemic. Journal of the American College of Surgeons 2020. https://doi.org/10.1016/j.jamcollsurg.2020.04.011

15.Jayasinghe R, Jayarajah U, Seneviratne S: Consensus on perioperative surgical practice during the COVID-19 pandemic: an appraisal of the literature. Sri Lanka Journal of Surgery 2020, 38 (2):57-61. https://doi.org/10.4038/sljs.v38i2.8738

16.Jayasinghe R, Jayarajah U, Seneviratne S: Consensus in Surgical Practice during the COVID-19 Pandemic: An Appraisal of the Literature. 2020.

17.Rational use of personal protective equipment for coronavirus disease 2019 (COVID-19)

[https://apps.who.int/iris/bitstream/handle/10665/331215/WHO2019-nCov-IPCPPE_use-2020.1-eng.pdf.]

18.Kumar S, Palta S, Saroa R, Mitra S: Anesthesiologist and COVID-19 - current perspective. Journal of Anaesthesiology Clinical Pharmacology 2020, 36(5):50.

https://doi.org/10.4103/joacp.joacp_205_20

19.Cook TM, El-Boghdadly K, McGuire B, McNarry AF, Patel A, Higgs $\mathrm{A}$ : Consensus guidelines for managing the airway in patients with COVID-19: Guidelines from the Difficult Airway Society, the Association of Anaesthetists the Intensive Care Society, the Faculty of Intensive Care Medicine and the Royal College of Anaesthetists. Anaesthesia 2020, 75(6):785-799. https://doi.org/10.1111/anae.15054

20.Brown J, Gregson FKA, Shrimpton A, Cook TM, Bzdek BR, Reid JP, Pickering AE: A quantitative evaluation of aerosol generation during tracheal intubation and extubation. Anaesthesia 2021, 76(2):174-181. https://doi.org/10.1111/anae.15292

21.College of Anaesthesiologists and Intensivists of Sri Lanka, Interim guidelines on management of critically ill patients with COVID-19 [https://anaesthesia.lk/sdm_downloads/interimguidelines-on-management-of-critically-ill-patients-withcovid-19/]

22.Sorbello M, Rosenblatt W, Hofmeyr R, Greif R, Urdaneta F: Aerosol boxes and barrier enclosures for airway management in COVID-19 patients: a scoping review and narrative synthesis. BJA: British Journal of Anaesthesia 2020, 125(6):880. https://doi.org/10.1016/j.bja.2020.08.038

23. Velly L, Gayat E, Quintard H, Weiss E, De Jong A, Cuvillon P, Audibert G, Amour J, Beaussier M, Biais M: Guidelines: Anaesthesia in the context of COVID-19 pandemic. Anaesthesia Critical Care \& Pain Medicine 2020, 39(3):395-415. https://doi.org/10.1016/j.accpm.2020.05.012

24.Interim Infection Prevention and Control Recommendations for Patients with Suspected or Confirmed Coronavirus Disease 2019 (COVID-19) in Healthcare Settings

[https://www.cdc.gov/coronavirus/2019-ncov/infectioncontrol/control-recommendations.html?CDC_AA_refVal= https $\% 3 \mathrm{~A} \% 2 \mathrm{~F} \% 2 \mathrm{Fwww} . \mathrm{cdc}$.gov\%2Fcoronavirus $\% 2 \mathrm{~F} 2019$ ncov $\% 2$ Fhcp $\% 2$ Finfection-control.html]

25.Malhotra N, Bajwa SJS, Joshi M, Mehdiratta L, Trikha A: COVID operation theatre-advisory and position statement of Indian Society of Anaesthesiologists (ISA National). Indian Journal of Anaesthesia 2020, 64(5):355.

https://doi.org/10.4103/ija.ija_288_20

26.Prakash L, Dhar SA, Mushtaq M: COVID-19 in the operating room: a review of evolving safety protocols. Patient Safety in Surgery 2020, 14(1):30.

https://doi.org/10.1186/s13037-020-00254-6 